\title{
Ocular comfort assessment of lifitegrast ophthalmic solution $5.0 \%$ in OPUS-3, a Phase III randomized controlled trial
}

This article was published in the following Dove Press journal:

Clinical Ophthalmology

\author{
Kelly K Nichols' \\ Edward Holland ${ }^{2}$ \\ Melissa M Toyos ${ }^{3}$ \\ James $\mathrm{H} \mathrm{Peace}^{4}$ \\ Parag Majmudar ${ }^{5}$ \\ Aparna Raychaudhuri ${ }^{6}$ \\ Mohamed Hamdani ${ }^{6}$ \\ Monica Roy ${ }^{6}$ \\ Amir Shojaei ${ }^{6}$ \\ 'School of Optometry, University \\ of Alabama at Birmingham, \\ Birmingham, AL, ${ }^{2}$ Cincinnati Eye \\ Institute, Edgewood, KY, ${ }^{3}$ Toyos \\ Clinic, Nashville, TN, ${ }^{4}$ United Medical \\ Research Institute, Inglewood, CA, \\ ${ }^{5}$ Chicago Cornea Consultants, Ltd., \\ Hoffman Estates, IL, ${ }^{6}$ Shire, Lexington, \\ MA, USA
}

Purpose: To evaluate ocular comfort of lifitegrast ophthalmic solution 5.0\% among patients with dry eye disease (DED) in the OPUS-3 trial.

Methods: OPUS-3 was a multicenter, randomized, double-masked, placebo-controlled study. Adults with DED and recent artificial tear use were randomized 1:1 (lifitegrast:placebo) to ophthalmic drops twice daily for 84 days. On days 0 (baseline), 14, 42, and 84, drop comfort score (scale, $0-10 ; 0=$ very comfortable, $10=$ very uncomfortable) was measured at $0,1,2$, and 3 minutes postinstillation. If the score was $>3$ at 3 minutes, assessment was repeated at 5,10 , and 15 minutes until score $\leq 3$. Ocular treatment-emergent adverse events (TEAEs) were assessed.

Results: Overall, 711 participants were randomized ( $n=357$ received lifitegrast; $n=354$ received placebo). Drop comfort scores for lifitegrast-treated participants improved within 3 minutes of instillation (mean scores on day 84 for both study and fellow eyes: instillation: lifitegrast, 3.4, placebo, 1.0; 3 minutes: lifitegrast, 1.5 , placebo, 0.7$)$. The majority (64\%-66\%) of participants had scores $<3$ within 3 minutes postinstillation on days 14, 42, and 84 . In participants with scores $>3$ at 3 minutes, the mean score in the lifitegrast group was similar to or better than that in the placebo group at 5,10 , or 15 minutes postinstillation. Lifitegrast appeared to be well tolerated, with ocular TEAEs rarely leading to discontinuation.

Conclusion: In OPUS-3, lifitegrast appeared to be well tolerated and drop comfort scores approached placebo levels by 3 minutes postinstillation.

Keywords: drop comfort, dry eye disease, lifitegrast ophthalmic solution 5.0\%, ocular comfort, OPUS-3

\section{Introduction}

Dry eye disease (DED) is a common, multifactorial disease of the ocular surface that is characterized by a loss of homeostasis of the tear film and can lead to ocular symptoms. Despite numerous candidates being investigated for the treatment of DED, no topical therapeutics had been approved in the United States since cyclosporine ophthalmic emulsion (Restasis ${ }^{\circledR}$; Allergan, Irvine, CA, USA) in 2003. In July 2016, lifitegrast ophthalmic solution 5.0\% (Xiidra ${ }^{\circledR}$; Shire, Lexington, MA, USA) was approved in the United States for the treatment of the signs and symptoms of DED, and it is the first in a new class of drugs called lymphocyte function-associated antigen 1 (LFA-1) antagonists. Lifitegrast blocks the interaction between cell surface proteins, LFA-1, and intercellular adhesion molecule 1. Evidence suggests that inhibition of LFA-1/ intercellular adhesion molecule 1 interaction interrupts the inflammatory cascade associated with DED. ${ }^{2}$
Correspondence: Amir Shojaei

Shire, 300 Shire Way, Lexington, MA 0242I, USA

Tel +l 4845958787

Email ashojaei@shire.com 
The clinical program supporting US Food and Drug Administration approval of lifitegrast comprised five randomized controlled trials: one Phase II efficacy/safety trial, ${ }^{3}$ three Phase III efficacy/safety trials, ${ }^{4-6}$ and a 1-year safety study. ${ }^{7}$ In the most recently completed Phase III study, OPUS-3, lifitegrast significantly improved symptoms of eye dryness, as measured by eye dryness score (EDS), compared with placebo in participants with DED. ${ }^{4}$ Consistent with previous lifitegrast trials, the drug was generally well tolerated in OPUS-3, and no serious ocular treatment-emergent adverse events (TEAEs) occurred. ${ }^{4}$ Ocular TEAEs in OPUS-3 were reported previously. ${ }^{4}$

Comfort upon instillation and ocular tolerability in general influence patient preference and therefore effectiveness of prescribed ophthalmic treatments. ${ }^{8}{ }^{89}$ In this analysis, we report drop comfort score of lifitegrast in the context of the overall assessment of ocular tolerability in OPUS-3.

\section{Methods}

\section{Study design and participants}

OPUS-3 (NCT02284516) was a prospective, multicenter, randomized, double-masked, placebo-controlled study conducted in the United States. The study followed the tenets of the Declaration of Helsinki; before study initiation, the protocol and informed consent form were reviewed and approved by the appropriate ethics committee or institutional review board (IRB) for each site. The ethics committees/IRBs were Alpha IRB (San Clemente, CA, USA), Western IRB (Puyallup, WA, USA), Sterling IRB (Atlanta, GA, USA), and the University of Houston (Houston, TX, USA). All participants provided written informed consent.
Study design and participant details have been previously described in full. ${ }^{4}$ Briefly, adults with a history of self-reported DED, Schirmer Tear Test score (without anesthesia) $\geq 1$ and $\leq 10 \mathrm{~mm}$, EDS score (visual analog scale, $0-100$; single score for both eyes) $\geq 40$, corneal staining score $\geq 2.0$ in one or more regions in one or more eyes (scale, $0-4$; 0 = none, 4 = coalescent; with 0.5 -point increments), and a history of artificial tear use within 30 days of study entry were randomized 1:1 (lifitegrast:placebo) to ophthalmic drops twice daily for 84 days.

Randomization was stratified by baseline inferior corneal fluorescein staining score $(\leq 1.5$ or $>1.5)$ in the study eye and EDS score $(<60$ or $\geq 60)$ to ensure balance between the treatment groups. The study comprised five visits: screening on visits 1 (day-14) and 2 (day 0, baseline) and efficacy/ safety assessments on visits 3 (day 14), 4 (day 42), and 5 (day 84; Figure 1). Participants who met eligibility criteria at the end of visit 1 received twice-daily open-label placebo for $\sim 2$ weeks. Prohibited medications during the study were topical cyclosporine or any other ophthalmic medication, including artificial tears, antihistamines, corticosteroids, or mast cell stabilizers.

\section{Drop comfort assessment}

Initial drop comfort of randomized treatment for both the study and fellow eyes was evaluated at visits $2-5$ (days 0 , 14,42 , and 84) using participant-reported assessments on a $0-10$ scale $\left(0=\right.$ very comfortable, $10=$ very uncomfortable $\left.{ }^{10}\right)$. The assessment was made immediately after instillation of the drop ( 0 minutes), and at 1,2, and 3 minutes postinstillation at the site by trained study personnel. We defined

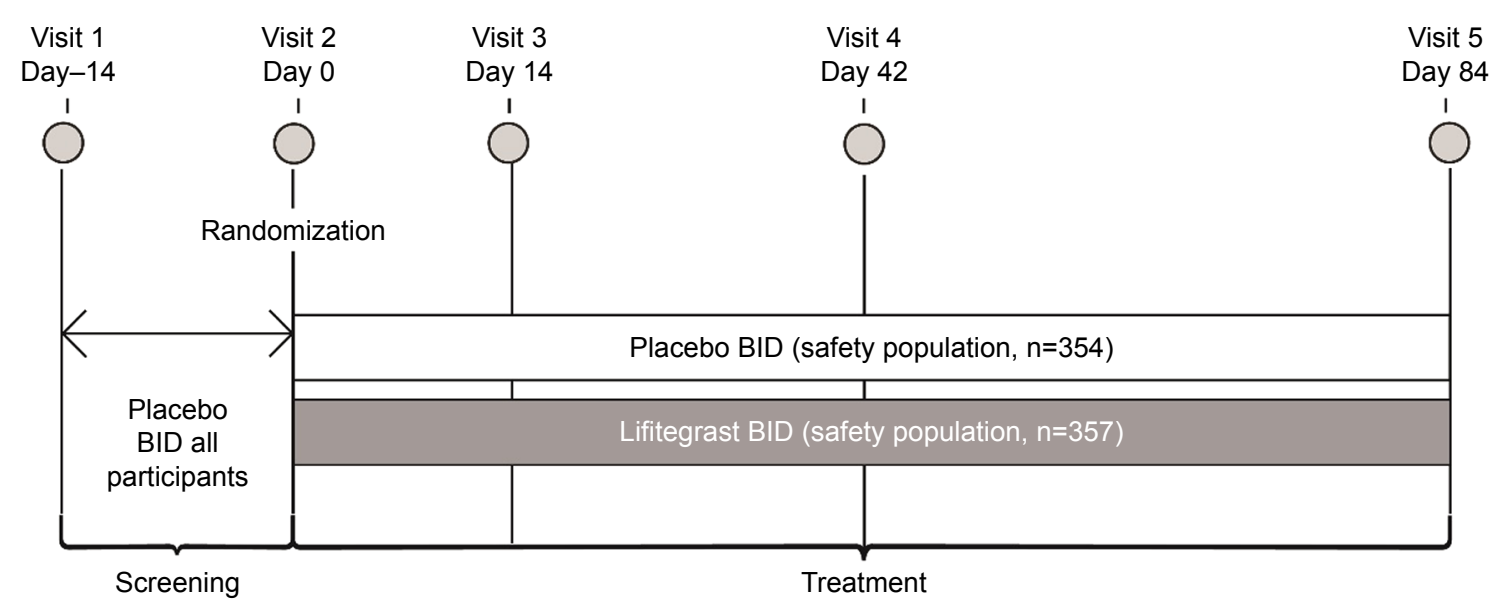

Figure I Study design of OPUS-3.

Notes: Drop comfort was evaluated on days $0,14,42$, and 84 with participant-reported assessments on a $0-10$ scale $(0=$ very comfortable, $10=$ very uncomfortable). All AEs were collected from the time the participant signed informed consent through the last study visit (day 84 or early termination).

Abbreviations: AE, adverse event; BID, twice daily. 
drop discomfort as drop comfort score $>3$, consistent with prior lifitegrast trials. If drop comfort score was not $\leq 3$ at 3 minutes postinstillation, the assessment was repeated at 5 , 10 , and 15 minutes, as needed, until the score was $\leq 3$. If the score was $>3$ at 15 minutes, it was recorded as an adverse event (AE). Such events were coded under either instillation site reaction or instillation site irritation, depending on whether drop comfort score $>3$ was because of ocular blurriness or ocular burning, respectively. Per the trial protocol, hypothesis testing was not performed for drop comfort or any other safety measures.

\section{Ocular tolerability assessment}

AEs recorded after starting randomized treatment were considered TEAEs. The investigators assessed AEs for severity (mild, moderate, and severe), for relationship to randomized treatment and seriousness. All AEs were coded using the Medical Dictionary for Regulatory Activities (MedDRA; MedDRA MSSO version 14.1; McLean, VA, USA).

A number of Verbatim Terms featuring ocular burning upon instillation of study drug were coded to the MedDRA Preferred Term instillation site irritation. Verbatim terms involving blurred/blurry vision or ocular discomfort were coded to the Preferred Term instillation site reaction. The Verbatim Terms of decreased visual acuity and decreased vision per best-corrected visual acuity (BCVA) were coded to the Preferred Term visual acuity reduced. Participants may have experienced one or more ocular TEAEs, which may or may not have led to discontinuation. Visual acuity was assessed using an Early Treatment Diabetic Retinopathy Study chart with a light box at each study visit. At visit 5 (day 84), BCVA (logarithm of the minimum angle of resolution $[\log M A R])$ was assessed before and after the final treatment. Clinically significant changes in visual acuity were recorded as AEs.

\section{Results}

\section{Participants}

A total of 711 participants were randomized (lifitegrast, $\mathrm{n}=355$; placebo, $\mathrm{n}=356$ ) and comprised the randomized and intention-to-treat populations. The safety population (lifitegrast, $n=357$; placebo, $n=354$ ) consisted of participants who received one or more treatment doses and was based on the treatment received.

Demographics (Table 1) and baseline DED severity characteristics were balanced between treatment groups (data previously reported ${ }^{4}$ ). Mean (standard deviation [SD]) inferior corneal fluorescein staining score at baseline for the
Table I Demographic and baseline characteristics (randomized population)

\begin{tabular}{|c|c|c|c|}
\hline Characteristic & $\begin{array}{l}\text { PBO, } \\
n=356\end{array}$ & $\begin{array}{l}\text { LIF, } \\
\mathrm{n}=355\end{array}$ & $\begin{array}{l}\text { Total, } \\
\mathrm{n}=7 \mathrm{II}\end{array}$ \\
\hline Mean (SD) age, years & $58.6(14.84)$ & $58.8(14.10)$ & $58.7(14.47)$ \\
\hline$>65, \mathrm{n}(\%)$ & $137(38.5)$ & $128(36.1)$ & $265(37.3)$ \\
\hline$>75, \mathrm{n}(\%)$ & $44(12.4)$ & $48(13.5)$ & $92(12.9)$ \\
\hline Female, n (\%) & $269(75.6)$ & $268(75.5)$ & $537(75.5)$ \\
\hline \multicolumn{4}{|l|}{ Ethnicity, n (\%) } \\
\hline Hispanic or Latino & $58(16.3)$ & $60(16.9)$ & $118(16.6)$ \\
\hline Not Hispanic or Latino & $298(83.7)$ & $295(83.1)$ & $593(83.4)$ \\
\hline \multicolumn{4}{|l|}{ Race, n (\%) } \\
\hline $\begin{array}{l}\text { American Indian or } \\
\text { Alaskan native }\end{array}$ & 0 & $2(0.6)$ & $2(0.3)$ \\
\hline Asian & $24(6.7)$ & $24(6.8)$ & $48(6.8)$ \\
\hline Black or African American & $47(13.2)$ & $48(13.5)$ & $95(13.4)$ \\
\hline Native Hawaiian or other & $\mathrm{I}(0.3)$ & $2(0.6)$ & $3(0.4)$ \\
\hline \multicolumn{4}{|l|}{ Pacific Islander } \\
\hline White & $279(78.4)$ & $265(74.6)$ & $544(76.5)$ \\
\hline Other & $5(1.4)$ & $14(3.9)$ & $19(2.7)$ \\
\hline Mean (SD) baseline EDS score & $69.0(17.08)$ & $68.3(16.88)$ & - \\
\hline $\begin{array}{l}\text { Mean (SD) baseline ICSS score } \\
\text { (study eye) }\end{array}$ & $2.46(0.744)$ & $2.46(0.684)$ & - \\
\hline $\begin{array}{l}\text { Mean (SD) baseline STT score } \\
\text { (study eye) }\end{array}$ & $5.18(2.697)$ & $4.98(2.647)$ & - \\
\hline
\end{tabular}

Notes: Two participants were randomized to the PBO group but incorrectly received LIF. These participants were included in the LIF group for the safety population, but in the $\mathrm{PBO}$ group for the randomized and intention-to-treat populations. Adapted from Holland EJ, Luchs J, Karpecki PM, et al. Lifitegrast for the treatment of dry eye disease: results of a phase III, randomized, double-masked, placebo-controlled trial (OPUS-3). Ophthalmology. 2017;124(I):53-60.4

Abbreviations: EDS, eye dryness score; ICSS, inferior corneal fluorescein staining score; LIF, lifitegrast; PBO, placebo; SD, standard deviation; STT, Schirmer Tear Test.

study eye was $2.46(0.744)$ in the placebo group and 2.46 (0.684) in the lifitegrast group; mean (SD) EDS score was 69.0 (17.08) and 68.3 (16.88), respectively.

\section{Drop comfort}

For all visits, over the first 3 minutes postinstillation, mean drop comfort score of participants in the placebo group was lower (more comfortable) than that in the lifitegrast group. However, drop comfort score for participants treated with lifitegrast improved within 3 minutes of instillation. Numeric improvements were also observed across visits (days 0-84; Figure 2). The majority $(64 \%-66 \%)$ of participants in the lifitegrast group had drop comfort scores $<3$ within 3 minutes postinstillation on days 14, 42, and 84 (Figure 3).

In participants who were reassessed for drop comfort at 5 , 10 , or 15 minutes postinstillation, mean score in the lifitegrast group was similar to or better than that in the placebo group at these time points (Figure 4). In both groups, the proportion of participants experiencing drop discomfort (score $>3$ ), and subsequently reassessed, decreased over time at each postinstillation time point. For example, at day 0, 10\% (36/354), 

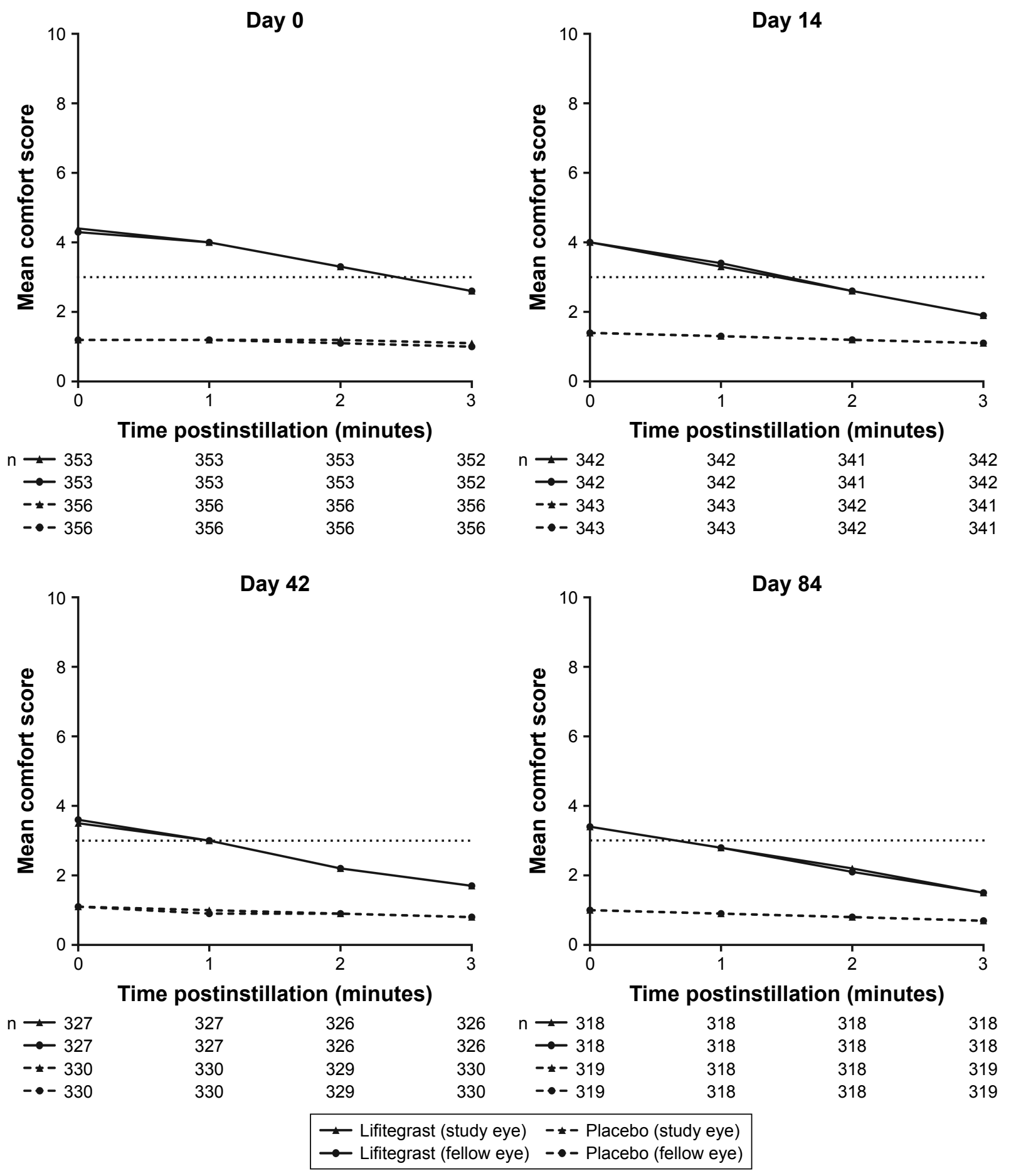

Figure 2 Drop comfort scores at 0-3 minutes postinstillation (safety population).

Notes: Drop comfort score (scale, 0-10; $0=$ very comfortable, $10=$ very uncomfortable); drop discomfort corresponds to scores $>3$. Scores between the study eye and fellow eye overlap.

and $27 \%$ (98/357) of participants in the placebo and lifitegrast groups, respectively, had study eye scores reassessed for drop comfort at 5 minutes postinstillation compared with $7 \%$ (24/354) and $11 \%$ (41/357) of participants, respectively, at 15 minutes postinstillation (Figure 3; see participant numbers above bars). The proportions of participants experiencing drop discomfort also decreased across visits, that is, at day 0 , $7 \%$ (24/354), and $11 \%$ (41/357) of participants in the placebo and lifitegrast groups, respectively, had study eye scores reassessed for drop comfort at 15 minutes postinstillation compared with 3\% (10/354) and 5\% (17/357), respectively, at day 84 (Figure 3). 


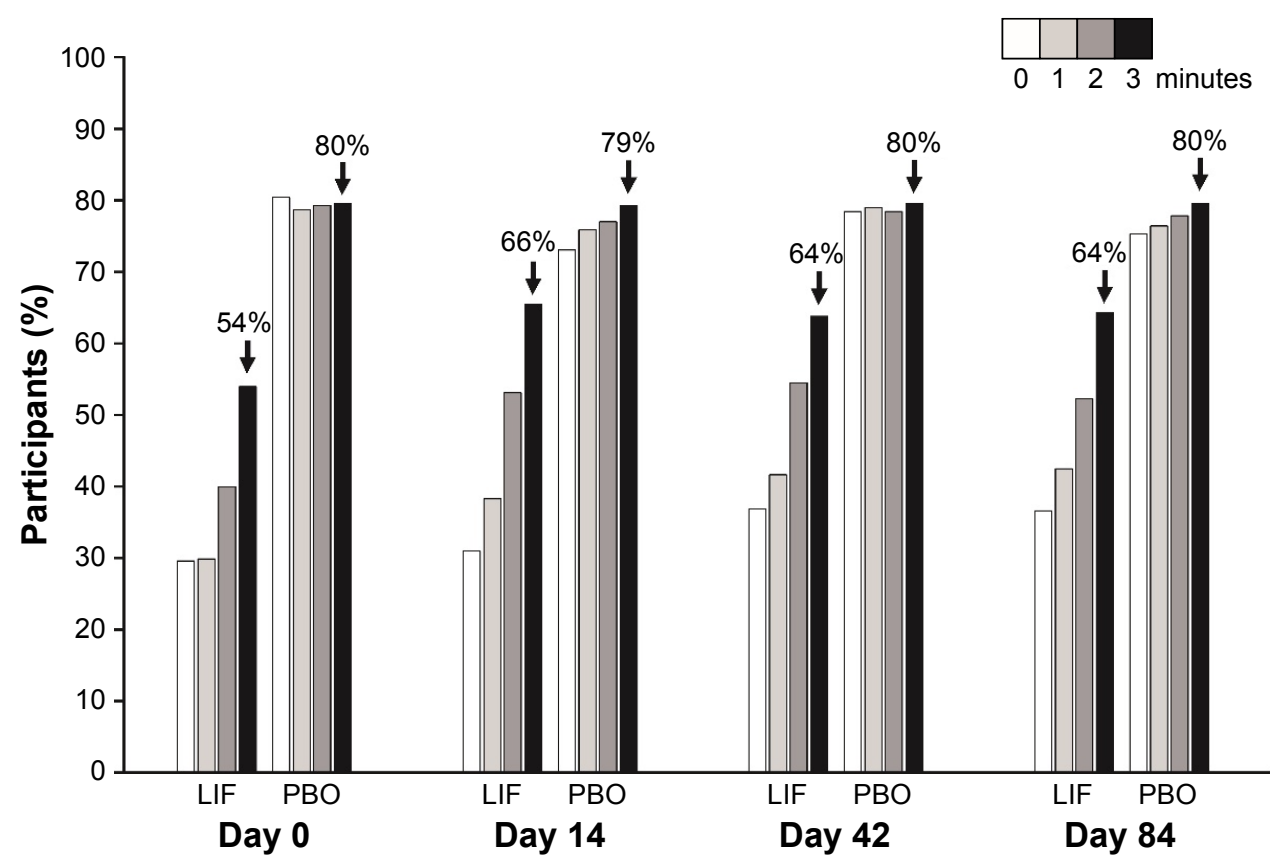

Figure 3 Participants with drop comfort score $<3$ by visit (both eyes; safety population).

Notes: Safety population in each treatment group was used as a denominator in percentage calculation (lifitegrast, $\mathrm{n}=357$; $\mathrm{placebo}, \mathrm{n}=354$ ). Drop comfort score (scale, 0-10; $0=$ very comfortable, $10=$ very uncomfortable).

Abbreviations: LIF, lifitegrast; PBO, placebo.

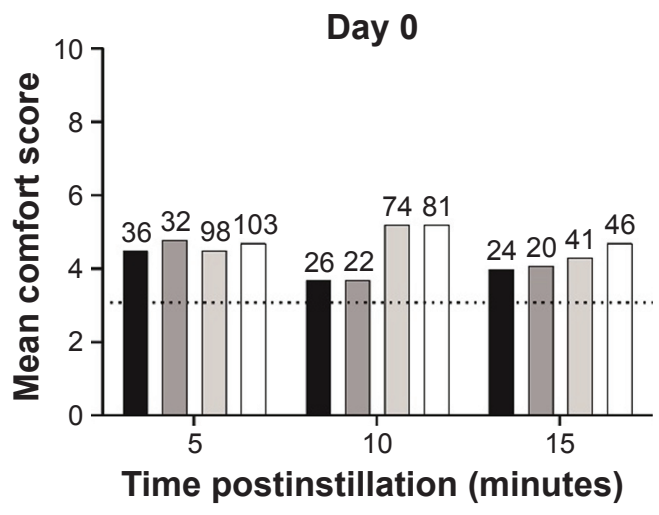

Day 42

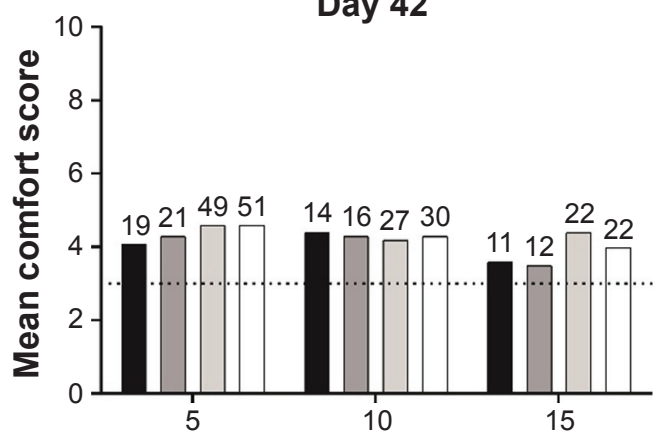

Time postinstillation (minutes)

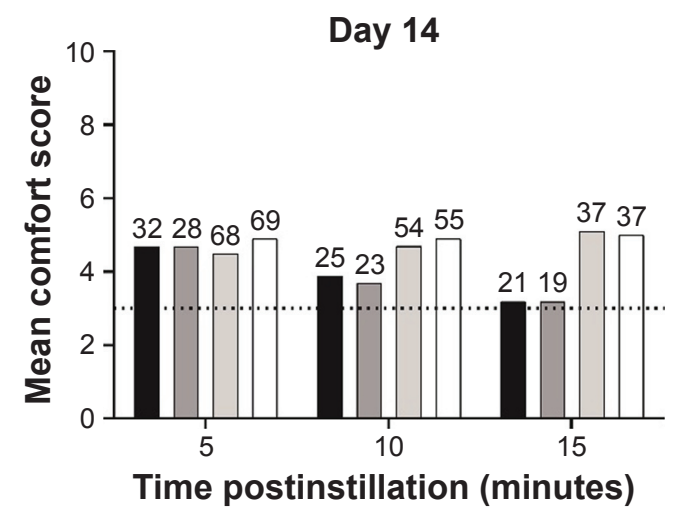

Day 84

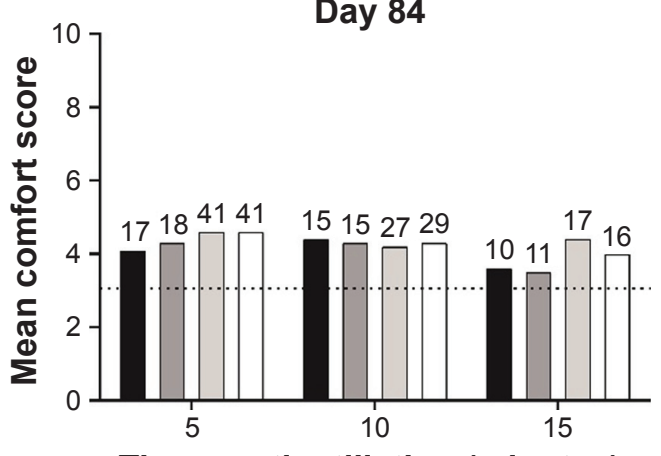

Time postinstillation (minutes)

\section{$\square$ Lifitegrast (study eye) $\square$ Placebo (study eye) Lifitegrast (fellow eye) $\square$ Placebo (fellow eye)}

Figure 4 Drop comfort scores at 5, 10, and 15 minutes postinstillation among participants with drop comfort score $>3$ at 3-minute assessment (safety population; numbers above bars indicate participant numbers).

Note: Drop comfort score (scale, 0-10; 0 = very comfortable, 10 = very uncomfortable); drop discomfort corresponds to scores $>3$. 
Table 2 Demographics and baseline characteristics of participants with drop comfort score $>3$ in the study eye at $\geq 15$ minutes postinstillation (safety population)

\begin{tabular}{|c|c|c|c|}
\hline Characteristic & $\begin{array}{l}\text { PBO, } \\
n=16\end{array}$ & $\begin{array}{l}\text { LIF, } \\
n=26\end{array}$ & $\begin{array}{l}\text { Total, } \\
\mathrm{n}=42\end{array}$ \\
\hline Mean (SD) age, years & $58.6(9.55)$ & $63.8(11.04)$ & $61.8(10.70)$ \\
\hline$>65, \mathrm{n}(\%)$ & $5(3 \mid .3)$ & II (42.3) & $16(38.1)$ \\
\hline$>75, \mathrm{n}(\%)$ & 0 & $7(26.9)$ & $7(16.7)$ \\
\hline Female, n (\%) & II (68.8) & $20(76.9)$ & $31(73.8)$ \\
\hline \multicolumn{4}{|l|}{ Ethnicity, n (\%) } \\
\hline Hispanic or Latino & $7(43.8)$ & $6(23.1)$ & $13(3 \mid .0)$ \\
\hline Not Hispanic or Latino & $9(56.3)$ & $20(76.9)$ & $29(69.0)$ \\
\hline \multicolumn{4}{|l|}{ Race, n (\%) } \\
\hline Asian & $2(12.5)$ & $5(19.2)$ & $7(16.7)$ \\
\hline Black or African American & $\mathrm{I}(6.3)$ & $2(7.7)$ & $3(7.1)$ \\
\hline Native Hawaiian or other & 0 & I (3.8) & $\mathrm{I}(2.4)$ \\
\hline \multicolumn{4}{|l|}{ Pacific Islander } \\
\hline White & $12(75.0)$ & $15(57.7)$ & $27(64.3)$ \\
\hline Other & $\mathrm{I}(6.3)$ & $3(11.5)$ & $4(9.5)$ \\
\hline Mean (SD) baseline EDS score & $71.8(19.08)$ & $69.0(16.14)$ & - \\
\hline $\begin{array}{l}\text { Mean (SD) baseline ICSS score } \\
\text { (study eye) }\end{array}$ & $2.59(0.935)$ & $2.44(0.739)$ & - \\
\hline $\begin{array}{l}\text { Mean (SD) baseline STT score } \\
\text { (study eye) }\end{array}$ & $6.19(2.880)$ & $4.65(2.097)$ & - \\
\hline
\end{tabular}

Abbreviations: EDS, eye dryness score; ICSS, inferior corneal fluorescein staining score; LIF, lifitegrast; PBO, placebo; SD, standard deviation; STT, Schirmer Tear Test.

The demographic and baseline characteristics of participants with drop discomfort in the study eye at $\geq 15$ minutes postinstillation (Table 2) were generally similar to those of the overall study population except for marginally higher rates of older and Asian participants.

\section{Ocular tolerability}

As described previously, ${ }^{4}$ there were no serious ocular TEAEs reported in OPUS-3. The most common ocular TEAEs ( $>5 \%$ of participants in either treatment group) were instillation site irritation and instillation site reaction, which included occurrences of drop comfort score $>3$ at 15 minutes. All cases of instillation site irritation and instillation site reaction were mild to moderate in severity (Figure 5).

Discontinuations resulting from instillation site reaction and instillation site irritation were infrequent (five and four participants, respectively, in the lifitegrast group; two and zero participants, respectively, in the placebo group). Mean BCVA did not worsen over time in either treatment group; minimal changes between visits in visual acuity were similar between treatment groups (mean [SD] BCVA logMAR: day 0: lifitegrast, 0.063 [0.1278] vs placebo, 0.077 [0.1469]; day 84: lifitegrast, 0.049 [0.1167] vs placebo, 0.051 [0.1280]).

\section{Discussion}

While the efficacy of any ophthalmic drug is critical for its effectiveness, the comfort upon instillation and overall ocular tolerability is important for patient preference ${ }^{8}$ and may affect adherence to treatment. Thus, effectiveness and tolerability are likely linked in terms of patient compliance. A willingness-to-pay study conducted in 230 patients with glaucoma using topical treatment to lower intraocular pressure found that $72 \%$ and $85 \%$ of patients would pay more for an eye drop that did not cause stinging and blurring upon instillation, respectively. ${ }^{9}$

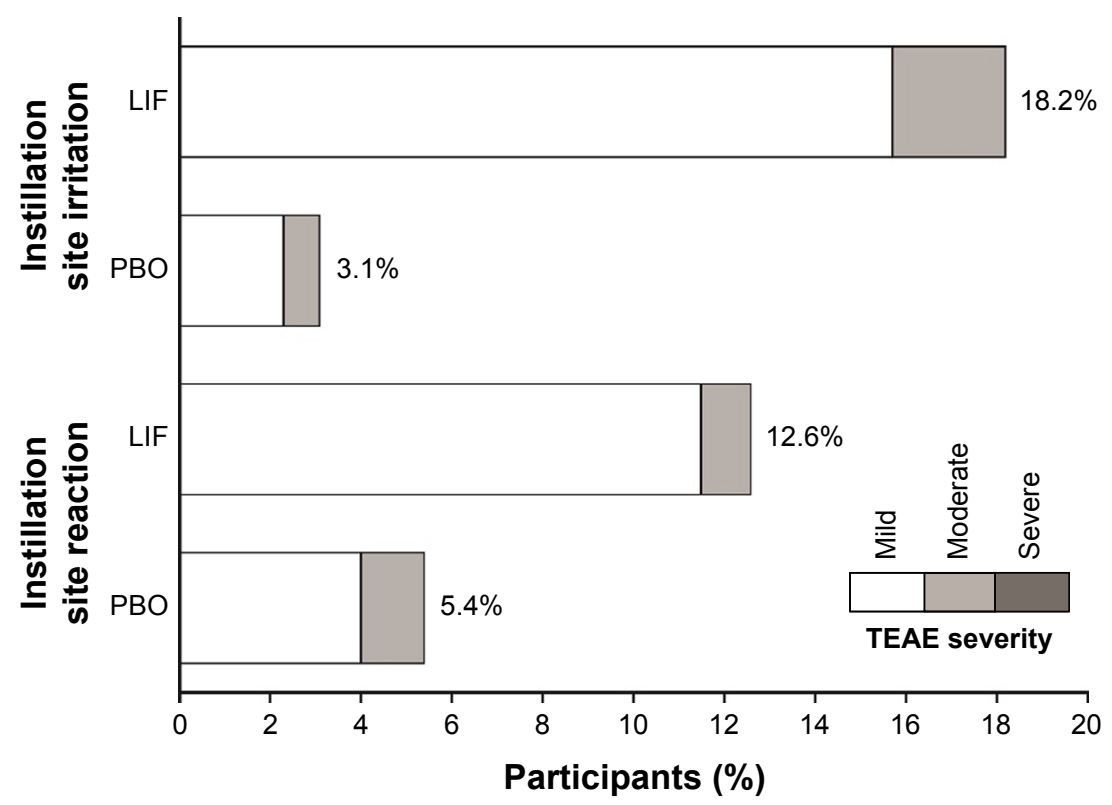

Figure 5 Incidence and severity of common ocular TEAEs (occurring in $>5 \%$ in either treatment group; safety population). Note: Verbatim Terms coding to instillation site irritation and instillation site reaction are described in the Methods section. Abbreviations: LIF, lifitegrast; PBO, placebo; TEAE, treatment-emergent adverse event. 
In this large, multicenter, randomized, controlled study, drop comfort scores with lifitegrast improved within 3 minutes of instillation, with scores approaching placebo levels, and the majority of participants reported drop comfort scores $<3$ at 3 minutes postinstillation at postbaseline visits where drop comfort was measured (days 14, 42, and 84). Consistent improvements in drop comfort were also observed over time, with mean drop comfort score $<3$ achieved more rapidly across visits. For participants with drop comfort scores $>3$ at 3 minutes, mean scores for the lifitegrast group were similar to or better than that for the placebo group at 5 , 10 , and 15 minutes.

We previously reported that in OPUS-3, lifitegrast was well tolerated, with no serious ocular TEAEs and a low rate $(<5 \%)$ of treatment discontinuation because of ocular TEAEs. ${ }^{4}$ The most common ( $>5 \%$ in either treatment group) ocular TEAEs in OPUS-3 were instillation site irritation and instillation site reaction, which were all mild to moderate in severity and rarely led to treatment discontinuation $(1.1 \%$ and $1.4 \%$ of participants treated with lifitegrast, respectively). In this analysis, we observed no deterioration in visual acuity following treatment with lifitegrast; $<1 \%$ of all participants (lifitegrast, 0.6\% [2/357]; placebo, 0.3\% [1/354]) experienced visual acuity reduced as an $\mathrm{AE}$.

The ocular comfort results in OPUS-3 are consistent with findings from previous 12-week (OPUS-1 and OPUS-2) (1,6 $^{5}$ and $1-$ year $^{7}$ trials in which patients with DED received twicedaily lifitegrast or placebo. In OPUS-3, the number of participants who experienced drop discomfort at $\geq 15$ minutes postinstillation was small ( $<10 \%$ in either treatment group). Notwithstanding the small number of these participants, their demographic and baseline characteristics were generally similar to the overall study population except for a marginally higher rate of older or Asian participants.

In our clinical trial and clinical care experiences, some participants with DED invariably experience ocular discomfort with all eye drops. Future studies to characterize this subset of patients would be useful from a clinical care and research perspective. In particular, the assessment of neuropathic ocular pain may be an important area of consideration. Recent research demonstrated that a subgroup of patients with DED who reported a higher number of comorbid chronic pain syndromes had features of neuropathic ocular pain and reported more severe DED symptoms than those with fewer comorbid conditions. ${ }^{11}$ In addition, patients with DED who experience increased tear film instability in combination with corneal hypersensitivity may be more susceptible to ocular discomfort. ${ }^{12-14}$ While it is unclear if these patients are unresponsive to treatment, the symptomatic profile demonstrates that these patients may be different from traditional patients with DED. In a Cochrane review of studies of artificial tear use in DED, $0 \%-55 \%$ of participants across the identified studies reported AEs, including significant symptoms and blurred vision. ${ }^{15}$ This suggests that a subset of patients show abnormal symptom profiles with drop use, warranting further consideration. Additional studies in symptomatic drop users, with either prescription drops or artificial tears, are needed to better characterize these patients and allow for improved care.

A limitation of our study is that it was not powered to compare drop comfort outcomes between treatment groups. Another limitation is that the use of MedDRA to code AEs may potentially lead to inaccuracies as a consequence of subjective application of codes.

\section{Conclusion}

In summary, in OPUS-3, twice-daily administration of lifitegrast in patients with DED appeared to be well tolerated, with instillation site AEs rarely leading to discontinuation and drop comfort scores approaching placebo levels by 3 minutes postinstillation.

\section{Acknowledgment}

OPUS-3 was sponsored by SARcode Bioscience (now a wholly owned subsidiary of Shire PLC) and Shire Development LLC. The authors thank Nasser Malik, PhD, CMPP, of Excel Scientific Solutions, who provided medical writing assistance, funded by SARcode Bioscience, a fully owned company of Shire PLC.

\section{Disclosure}

Kelly K Nichols has received research funding from Allergan, Bruder, Eleven Biotherapeutics, Kala, the National Institutes of Health, Shire PLC/SARcode, TearScience, and Vistakon, and has been a consultant for Allergan, InSite, Kala, Parion, Santen, ScienceBased Health, Shire PLC/SARcode, and Sun. Edward Holland has received research funding from Alcon, Allergan, Mati Therapeutics, Omeros, PRN, and Senju, and has been a consultant for Alcon, Allergan, Bausch \& Lomb, Kala, Mati Therapeutics, Omeros, PRN, RPS Diagnostics, Senju, Shire PLC/SARcode, TearLab, and TearScience. In addition, Dr Holland has been a speaker for Alcon, Allergan, Bausch \& Lomb, Omeros, Senju, Shire PLC/SARcode, and TearScience. Melissa Toyos has received research funding from Bausch \& Lomb/Valeant, DigiSight, INC Research, Ocular Therapeutix, Kala, Parexel, PRN, Senju, and Shire PLC, and has been a speaker for Alcon, Allergan, Bausch \& Lomb/Valeant, Shire PLC, and Sun. James H Peace has 
received research funding from and been a consultant for Shire PLC. Parag Majmudar has received research funding from Shire PLC, and has been a consultant for Allergan, Rapid Pathogen Screening, TearScience, and Valeant. Aparna Raychaudhuri and Monica Roy were employees of Shire PLC at the time of this work and own stock in Shire PLC. Mohamed Hamdani and Amir Shojaei are employees of Shire PLC and own stock/stock options in Shire. The authors report no other conflicts of interest in this work.

\section{References}

1. Craig JP, Nichols KK, Akpek EK, et al. TFOS DEWS II definition and classification report. Ocul Surf. 2017;15(3):276-283.

2. Perez VL, Pflugfelder SC, Zhang S, Shojaei A, Haque R. Lifitegrast, a novel integrin antagonist for treatment of dry eye disease. Ocul Surf. 2016;14(2):207-215.

3. Semba CP, Torkildsen GL, Lonsdale JD, et al. A phase 2 randomized, double-masked, placebo-controlled study of a novel integrin antagonist (SAR 1118) for the treatment of dry eye. Am J Ophthalmol. 2012; 153(6):1050.e1-1060.e1.

4. Holland EJ, Luchs J, Karpecki PM, et al. Lifitegrast for the treatment of dry eye disease: results of a phase III, randomized, double-masked, placebo-controlled trial (OPUS-3). Ophthalmology. 2017;124(1):53-60.

5. Sheppard JD, Torkildsen GL, Lonsdale JD, et al. Lifitegrast ophthalmic solution $5.0 \%$ for treatment of dry eye disease: results of the OPUS-1 phase 3 study. Ophthalmology. 2014;121(2):475-483.

6. Tauber J, Karpecki P, Latkany R, et al. Lifitegrast ophthalmic solution $5.0 \%$ versus placebo for treatment of dry eye disease: results of the randomized phase III OPUS-2 study. Ophthalmology. 2015;122(12): 2423-2431.
7. Donnenfeld ED, Karpecki PM, Majmudar PA, et al. Safety of lifitegrast ophthalmic solution $5.0 \%$ in patients with dry eye disease: a 1-year, multicenter, randomized, placebo-controlled study. Cornea. 2016;35(6): 741-748.

8. Leonardi A, Zafirakis P. Efficacy and comfort of olopatadine versus ketotifen ophthalmic solutions: a double-masked, environmental study of patient preference. Curr Med Res Opin. 2004;20(8):1167-1173.

9. Jampel HD, Schwartz GF, Robin AL, Abrams DA, Johnson E, Miller RB. Patient preferences for eye drop characteristics: a willingness-to-pay analysis. Arch Ophthalmol. 2003;121(4):540-546.

10. Torkildsen GL, Ousler GW 3rd, Gomes P. Ocular comfort and drying effects of three topical antihistamine/mast cell stabilizers in adults with allergic conjunctivitis: a randomized, double-masked crossover study. Clin Ther. 2008;30(7):1264-1271.

11. Galor A, Covington D, Levitt AE, et al. Neuropathic ocular pain due to dry eye is associated with multiple comorbid chronic pain syndromes. J Pain. 2016;17(3):310-318.

12. Begley C, Simpson T, Liu H, et al. Quantitative analysis of tear film fluorescence and discomfort during tear film instability and thinning. Invest Ophthalmol Vis Sci. 2013;54(4):2645-2653.

13. Liu H, Begley C, Chen M, et al. A link between tear instability and hyperosmolarity in dry eye. Invest Ophthalmol Vis Sci. 2009;50(8): 3671-3679.

14. Kaido M, Kawashima M, Ishida R, Tsubota K. Relationship of corneal pain sensitivity with dry eye symptoms in dry eye with short tear break-up time. Invest Ophthalmol Vis Sci. 2016;57(3):914-919.

15. Pucker AD, Ng SM, Nichols JJ. Over the counter (OTC) artificial tear drops for dry eye syndrome. Cochrane Database Syst Rev. 2016; 2:CD009729.
Clinical Ophthalmology

\section{Publish your work in this journal}

Clinical Ophthalmology is an international, peer-reviewed journal covering all subspecialties within ophthalmology. Key topics include: Optometry; Visual science; Pharmacology and drug therapy in eye diseases; Basic Sciences; Primary and Secondary eye care; Patient Safety and Quality of Care Improvements. This journal is indexed on Submit your manuscript here: http://www.dovepress.com/clinical-ophthalmology-journal

\section{Dovepress}

PubMed Central and CAS, and is the official journal of The Society of Clinical Ophthalmology (SCO). The manuscript management system is completely online and includes a very quick and fair peer-review system, which is all easy to use. Visit http://www.dovepress.com/ testimonials.php to read real quotes from published authors. 